\title{
Transport properties of negative muons in matter*
}

\author{
P. K. Haff ${ }^{\dagger}$ \\ Niels Bohr Institute, DK-2100, Copenhagen, Denmark \\ P. Vogel \\ California Institute of Technology, Pasadena, California 91125 \\ (Received 4 June 1976)
}

\begin{abstract}
In deriving a formula for atomic capture ratios involving negative muons, Daniel postulates a model leading to a muon energy spectrum of a different character from that indicated by a more complete analysis. In this Comment we emphasize the dependence of the energy spectrum on both inelastic and capture processes, and suggest several experiments which may distinguish between different theoretical models.
\end{abstract}

There has been considerable experimental interest in investigating the manner in which stopped muons are shared among the distinguishable constituents of mixtures and compounds., ${ }^{1,2}$ Correspondingly there have been theoretical efforts to describe and predict the "capture ratios" in terms of the fundamental muon-atom interactions. However, not all of these efforts have recognized that both capture and stopping cross sections must be combined in a consistent way in order to describe the capture process.

Consider a target composed of atoms of species 1 (atomic number $Z_{1}$, percent concentration $n_{1}$ ), and species $2\left(Z_{2}, n_{2}\right)$. To make the following points a bit clearer we suppose that we are dealing with an alloy, or a mixture of gases. The number of muons captured on species 1 may be described ${ }^{3}$ by the general expression

$$
\begin{aligned}
& N\left(n_{1}, n_{2} ; Z_{1}, Z_{2}\right) \\
& \quad=n_{1} \int_{0}^{\infty} P\left(n_{1}, n_{2} ; Z_{1}, Z_{2} ; E\right) \sigma\left(Z_{1}, E\right) d E,
\end{aligned}
$$

where $E$ is the muon energy. The function $\sigma\left(Z_{1}, E\right)$ is the capture cross section of element $Z_{1}$, and $P$ is the steady-state energy spectrum of muons in the target. The function $P$ in general depends upon the relative concentrations of the species and their identity. It satisfies an integral equation $^{3}$ which describes the equality between the number of particles entering an energy bin $E, E+d E$ by inelastic scattering from higher energies, and the number leaving that bin via inelastic scattering and direct atomic capture. Thus $P$ and $\sigma$ are not at all independent in general, and a correct treatment of muon capture necessarily involves treating both stopping and capture on the same footing.

The functions $P$ and $\sigma$ were calculated by $\mathrm{us}^{3}$ for a classical model of the muon-atom interaction, and the dependence of the capture ratio $N_{1} / N_{2}$ on $n_{1} / n_{2}$ and $Z_{1}, Z_{2}$ was studied. Over the energy region where the capture is likely to occur, we found approximately

$$
P \sim E
$$

and

$$
\sigma(Z, E) \sim a-\frac{b}{Z^{1 / 3} \ln (1.3 Z)} E,
$$

where $a$ and $b$ are constants independent of $Z$. Combining (1), (2), and (3) yields (for $n_{1}=n_{2}$ )

$$
\frac{N_{1}}{N_{2}} \simeq \frac{Z_{1}^{2 / 3}\left[\ln \left(1.3 Z_{1}\right)\right]^{2}}{Z_{2}^{2 / 3}\left[\ln \left(1.3 Z_{2}\right)\right]^{2}}
$$

Recently the alternative formula

$$
\frac{N_{1}}{N_{2}}=\frac{Z_{1}^{1 / 3} \ln \left(0.57 Z_{1}\right)}{Z_{2}^{1 / 3} \ln \left(0.57 Z_{2}\right)}
$$

has been proposed by Daniel. ${ }^{4}$ Like Eq. (4) it has its origin in a classical picture of the muon-atom interaction, but is derived under a more severe set of approximations. The most important consequence of these approximations is his use of a "white" energy spectrum $P(E)$. In fact, if Eq. (2) is replaced by

$$
P=\text { const }
$$

then Eqs. (1), (3), and (6) give

$$
\frac{N_{1}}{N_{2}}=\frac{Z_{1}^{1 / 3} \ln \left(1.3 Z_{1}\right)}{Z_{2}^{1 / 3} \ln \left(1.3 Z_{2}\right)},
$$

which is essentially the result (5). The different logarithmic factors in (5) and (7) arise from different treatment of electron velocities. Reference 4 uses the average velocity instead of the correctly weighted Fermi velocity.

A careful treatment of the classical model of muon capture leads, however, to expression (2), and not (6), for the energy spectrum. Consequently, Eq. (5), although providing a reasonably good fit to capture ratios ${ }^{5}$ in the metallic halides, does 
not follow from a more complete consideration of the model. If, indeed, a flat energy spectrum is necessary to attain agreement with the data, then such a shape must follow from the same arguments used to derive the capture cross sections, as both energy loss and capture itself are moderated by the same forces.

Since the energy spectrum $P$ and the capture cross section $\sigma$ appear folded together in the integral (1), it is not so easy to test the theoretical models against experimental results in a direct way. In general $P$ depends upon both capture and inelastic scattering cross sections of both species. However, there are several experimental conditions which would emphasize independently the effect of the spectrum shape and the form of the capture cross section. Such experiments ${ }^{6}$ would therefore be particularly useful in discriminating between various models and in suggesting theoretical developments.

For example, consider a dilute concentration of species 1 in species $2, n_{1} \ll n_{2}$. The atoms of species 2 then form a moderator, i.e., the muon energy spectrum is determined exclusively by 2 . A measurement of the number of captures on 1 would give

$$
\begin{aligned}
N_{1}\left(n_{1}, n_{2} ; Z_{1}, Z_{2}\right) & \\
= & n_{1} \int_{0}^{\infty} P\left(0,1 ; Z_{2} ; E\right) \sigma\left(Z_{1}, E\right) d E,
\end{aligned}
$$

where $P$ is independent of $Z_{1}$. If the identity of the impurity 1 is changed, while that of the moderator remains the same, then the dependence of $N_{1}$ upon $Z_{1}$ may be mapped out. Any differences observed between $N_{1}\left(n_{1}, n_{2} ; Z_{1}, Z_{2}\right)$ and $N_{1}\left(n_{1}, n_{2} ; Z_{1}^{\prime}, Z_{2}\right)$ can be attributed solely to differences in the capture cross sections, $\sigma\left(Z_{1}, E\right)$ and $\sigma\left(Z_{1}^{\prime}, E\right)$.

The conjugate experiment, in which the impurity is kept constant while the moderator is changed, is also interesting. In this case, any observed dependence of $N_{1}$ upon $Z_{2}$ must arise from differences in the distribution function $P$.

The other variable at our disposal is the concentration ratio $n_{1} / n_{2}$. The cross section $\sigma$, of course, does not depend on $n_{1} / n_{2}$. Thus, if $Z_{1}$ and $Z_{2}$ are kept constant, all variations in $N_{1}\left(n_{1}, n_{2} ; Z_{1}, Z_{2}\right)$, except the trivial $n_{1}$ scaling, arise from the variation in the energy spectrum.

It is expected that experiments on binary alloys or gas mixtures will lead to results more easily interpreted than experiments using chemical compounds as targets. In the latter case the atomic components are usually present in comparable quantities so that the energy spectra depend upon the capture cross sections of both species.

Measurements performed along the lines described above would be useful probes of the transport properties which determine the fate of slow muons in matter. A convincing experimental identification of both stopping and capture is important for its emphasis on the necessity of a consistent transport treatment of muon capture, as well as for the additional tests of theoretical models which it would provide. A detailed theoretical treatment, when supported by the appropriate data, of the basic stopping and capture features outlined above would both stimulate and facilitate attempts to understand the more complicated phenomena associated with chemical bonding and crystalline structure in solid targets. $\dagger$ Present address: Wright Nuclear Structure Laboratory, Yale University, New Haven, Conn. 06520.

*Work supported in part by ERDA AT[04-3]-63.

${ }^{1}$ L. I. Ponomarev, Ann. Rev. Nucl. Sci. 23, 395 (1973), and references therein.

${ }^{2}$ F. L. Mausner, R. A. Naumann, J. A. Monard, and N. S. Kaplan, Phys. Lett. 56B, 145 (1975).

${ }^{3}$ P. Vogel, P. K. Haff, V. Akylas, and A. Winther, Nucl. Phys. A 254, 445 (1975).
${ }^{4}$ H. Daniel, Phys. Rev. Lett. 35, 1649 (1975).

${ }^{5}$ Note, however, that the data used in Ref. 4 to compare with Eq. (5) are not all mutually consistent. For example, the capture ratio in $\mathrm{NaCl}$, measured by three quoted groups, spans five standard deviations.

${ }^{6} \mathrm{~A}$ preliminary discussion of possible muonic-atom experiments was given by P. K. Haff and P. Vogel, Cal Tech. Laboratory Report BAP-5, August, 1975 (unpublished). 